\title{
Nanomachining Graphene with Ion Irradiation
}

\author{
Jani Kotakoski ${ }^{1}$ and Ossi Lehtinen ${ }^{1}$ \\ ${ }^{1}$ Div. of Materials Physics, University of Helsinki, P.O. Box 42, 00014 Helsinki, Finland.
}

\begin{abstract}
We present molecular dynamics simulations using both empirical potentials (EP) and density functional theory (DFT) on ion irradiation of graphene. The comparison between the two methods shows that EP gives not only qualitatively but also quantitatively reasonable estimates of defect production during ion irradiation in carbon nanosystems. Ion irradiation is shown to give rise to a range of interesting defects e.g. single, double and triple vacancies, bond rotations, close-by Frenkel pairs and more complex defect structures. We show that the creation of these defects is related to the atomic processes upon the ion impact, and define the critical irradiation angles both for maximum damage and no penetration as a function of the ion mass.
\end{abstract}

\section{INTRODUCTION}

Graphene is the ultimately thin membrane - a chicken wire structure made from $s p^{2}$ hybridized carbon atoms - which has attracted enormous attention since its discovery [1], not least because of its unique electronic properties [2]. An important aspect in this regard is the effect of disorder, especially since graphene grown with the chemical vapor deposition (CVD) method is known to contain a large proportion of dislocations and disordered areas, although some of the seen defects may be a result from the processes in preparing and imaging the structure [3]. Ion irradiation is a good method for a systematic study of the role of the disorder on the properties of graphene, because the irradiation doses and hence the amount of disorder can be easily controlled. To estimate the ion-irradiation-induced disorder in graphene, Raman spectroscopy [4, 5], atomic force microscopy [5, 6], local mobility measurements [7] and atomic resolution scanning tunneling microscopy [8] have been used. However, the underlaying atomic scale mechanisms are not known. In fact, even the separation between the directly created damage on graphene and the damage caused to the substrate or secondary damage to graphene via backscattering from the substrate has not been studied. In this study, we simulate impacts of energetic ions onto a suspended graphene sheet to describe the atomic scale events during ion irradiation of this material.

\section{THEORY}

In order to evaluate the characteristic quantities from first principles, we employed density functional theory (DFT) molecular dynamics (MD) simulations with the plane-wave basis set code VASP [9] with projector augmented wave (PAW) potentials [10] to describe the core electrons, and the generalized gradient approximation (GGA) [11] for exchange and correlation. In order to validate the use of PAW in high energy impact simulations where atoms appear at close separations, we compared PAW calculations with all-electron calculations carried 
out with the simulation code DMOL [12]. Comparing the energy of a Ar-C dimer with both methods as a function of the dimer length showed that the PAW results are reasonable up to at least $500 \mathrm{eV}$. To estimate the displacement threshold $T_{d}$ for a carbon atom in pristine graphene, we determined the kinetic energy which is needed by the atom in order to escape from the structure - a quantity similar to the escape velocity required by a space shuttle in order to surpass Earth's gravity - by carrying out a set of MD simulations in which a certain $T$ was assigned to one of the atoms. Our result $\left(T_{d}=22.2 \pm 0.2 \mathrm{eV}\right)$ is similar to an earlier estimate $(23 \mathrm{eV})$ obtained with a less accurate tight binding model [13]. Next, we went on to estimate the kinetic energy needed by an ion to cause a displacement via a head-on collision with a carbon atom. For Ar, we obtained a result of $K_{\mathrm{Ar}}=32.74 \pm 0.15 \mathrm{eV}$. We also calculated the energy barrier for several noble gas ions ( $\mathrm{He}, \mathrm{Ne}, \mathrm{Ar}, \mathrm{Kr}, \mathrm{Xe}$ ) to penetrate a graphene sheet through the middle of a hexagon (see Figure 1).

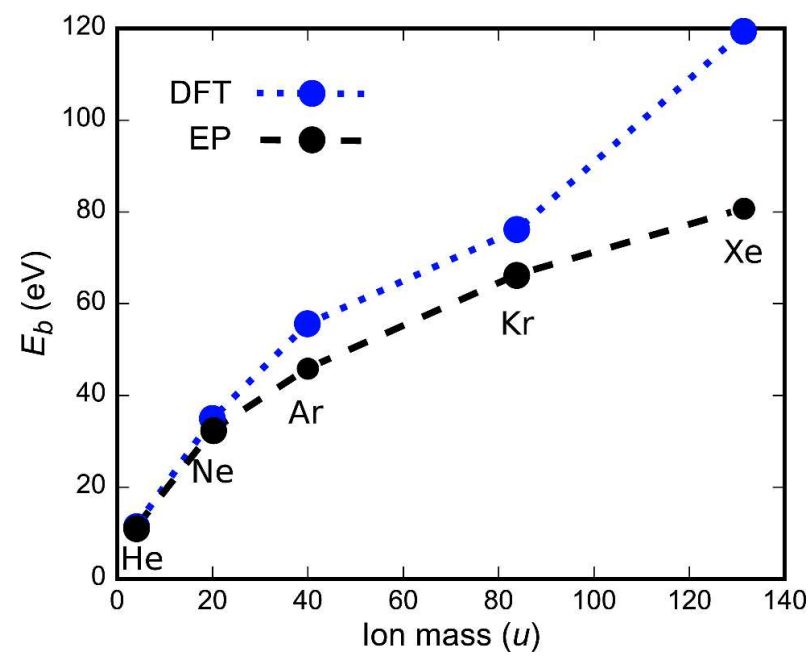

Figure 1. Energy barriers for penetrating a graphene layer through the middle of the hexagon for several noble gas ions as a function of the ion mass.

For the EP method simulations, we used a similar setup as in our earlier studies on ion irradiation of carbon nanotubes [14-18]. As the C-C interaction model, we employed the reactive analytical bond order potential by Brenner [19], whereas the interaction between the noble gas ions and carbon atoms was described with the universal repulsive potential by Ziegler, Biersack and Littmark (ZBL) [20]. Using this method, we obtained $T_{d}=22.05 \mathrm{eV}$ and $K_{\mathrm{Ar}}=32.33 \mathrm{eV}$ in a surprisingly good agreement with the above-presented DFT values. A comparison of the penetration energy barriers for EP and DFT is presented in Figure 1. Because the ZBL is a pair potential, this comparison displays the worst case estimate on the performance of the potential when compared to DFT calculations. In this light, the agreement is again surprisingly good. Based on the comparisons, the EP method gives not only qualitatively but also quantitatively reasonable estimates of defect production during ion irradiation in carbon nanosystems. For the irradiation simulations with the EP method, we used a target system consisting of 800 atoms, and modeled the dissipation of the kinetic energy brought in by an energetic ion through the Berendsen thermostat [21] at the borders of the system. A detailed description of the simulations of irradiation events with ion trajectory perpendicular to the graphene sheet can be found in Ref. [22]. We analyzed 150000 EP MD simulations to categorize all the irradiation-induced defects 
and calculated their probability of appearance as a function both of the momentum transferred from the ion to the lattice and of the recoil angle $\theta$ - angle of the velocity of the target atom (the one closest to the ion trajectory) with respect to the graphene plane, right after the impact (most of the damage is created by a carbon atom displaced close to the in-plane direction [22]). These probabilities are presented in Figure 2.

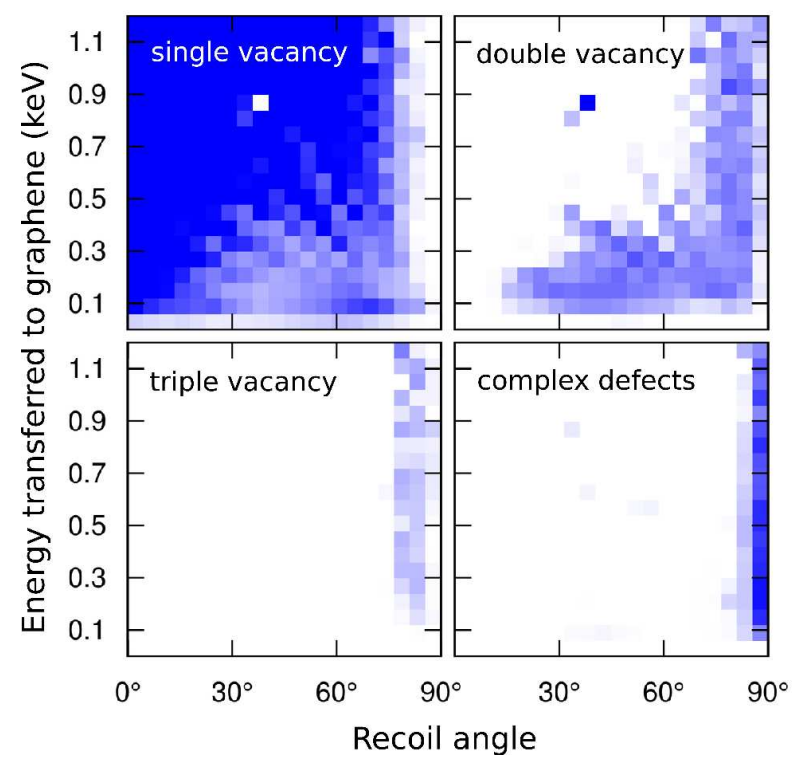

Figure 2. Probability $P$ for different defects to appear as function of the momentum transferred from ion to graphene and of scattering angle $\theta$ of the closest target atom with respect to the graphene sheet. Dark color denotes $P=1.0$, white is zero.

Following the same approach as in Ref. [22], we carried out another set of irradiation simulations, now with irradiation angles of $\Psi_{\text {ion }}$ in $[10,80]^{\circ}$ with respect to the graphene sheet. We again carefully analyzed the data to characterize the ion-irradiation-induced defects and noticed a systematic behavior for the $\Psi_{\text {ion }}$ which leads to maximum probability of the created complex defects as a function of the ion mass. This behavior is similar to the critical angle for ion channeling $\Psi_{c}$, for which a thorough theory has been developed [23]. A crude approximation for the critical angle is $\Psi_{c}=\left(E / K_{i o n}\right)^{1 / 2}$ where $E$ is an energy related to the barrier for the ion to penetrate the target material. For Ar irradiation of carbon nanotubes at energies above $1 \mathrm{keV}$, for channeling (i.e. reflecting from target creating no damage) $E=121.85 \mathrm{eV}$ has been obtained by fitting to data (note different units) [24]. From our data up to $1.5 \mathrm{keV}$, we were able to derive a general expression for $E$ for any ion. For channeling we obtained $E_{\mathrm{c}}=62 \mathrm{eV}$, and for submerging the ion to graphene we obtained $E_{\mathrm{d}}=83 \mathrm{eV}-(0.35 \mathrm{eV} / \mathrm{u}) m$, with an effective kinetic energy $K_{\text {ion }}^{\prime}$ $=K_{\text {ion }}-m^{2} /\left(400 \mathrm{u}^{2} / \mathrm{eV}\right)$, where $m$ is the ion mass in the unit of atomic mass (see Figure 3 ).

\section{DISCUSSION}

As can be seen from Figure 2, it is possible to distinguish two different mechanisms for defect creation under ion irradiation of graphene. For the simpler defects (single and double 
vacancies) there is a possibility of creating them directly with the impact of the ion, regardless of the angle to which the target atom scatters. However, for the higher order defects, the probability is close to zero for recoil angles away from the in-plane direction $\left(90^{\circ}\right)$. One needs to realize though that the situation changes when the ion trajectory is not perpendicular to the graphene sheet, since the ion will interact with a larger number of atoms. The decisive factor in damaging the target in this case is the scattering of the ion instead of that of the target atom (at higher energies the target atom will scatter in direction perpendicular to the ion trajectory [22]). Hence, it becomes important to know the critical angle for the ion to be scattered into the graphene plane. This process is similar to the channeling event, and consequently can be described in a similar manner, as we showed above.
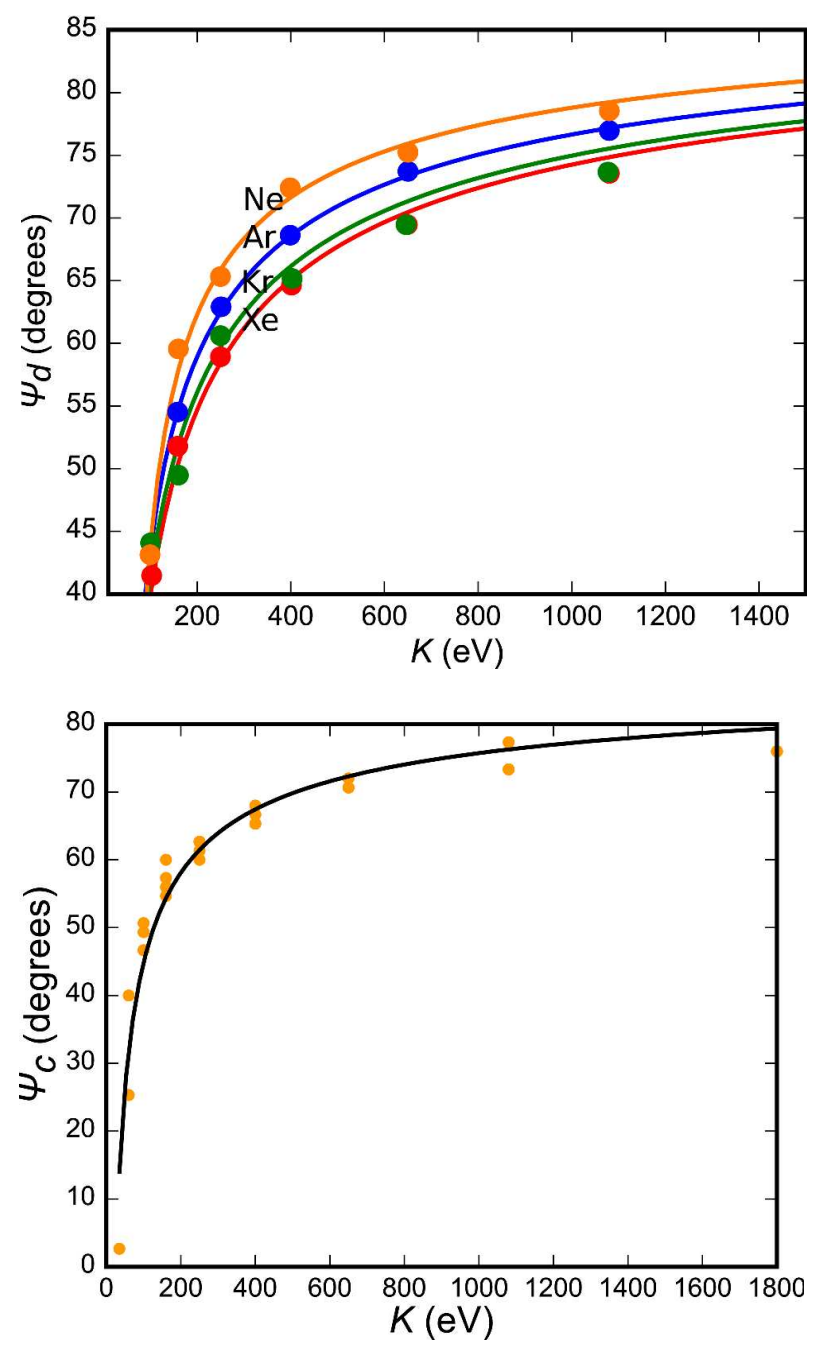

Figure 3. Critical irradiation angles for creating maximum damage $\Psi_{d}=\left(E_{d} / K^{\prime}{ }_{i o n}\right)^{1 / 2}$ and reflecting from the target $\Psi_{c}=\left(E / K_{i o n}\right)^{1 / 2}$ for different ions (see text).

\section{CONCLUSIONS}


In this study, we have combined density functional theory (DFT) calculations and empirical potentials (EP) method to perform molecular dynamics (MD) simulations on ion irradiation of graphene. We showed that EP gives not only qualitatively but also quantitatively reasonable estimates of defect production during ion irradiation in carbon nanosystems. We also show that the ion irradiation introduces a range of defects in graphene via complicated mechanisms related to scattering of the closest carbon atom and the scattering of the ion, depending on the irradiation angle. Finally, we derived an analytical expression for critical irradiation angles for the ion to both create maximum damage and to be reflected away from graphene without creating any damage.

\section{ACKNOWLEDGMENTS}

We wish to acknowledge the Finnish CSC in Espoo for computer time through the Grand Challenge program. This work was supported by the Academy of Finland through project DEMECAN.

\section{REFERENCES}

1. A. K. Geim and K. S. Novoselov, Nat Mater 6, 183 (2007).

2. A. H. Castro Neto, F. Guinea, N. M. R. Peres, K. S. Novoselov, and A. K. Geim, Rev. Mod. Phys. 81, 109 (2009).

3. H. Cao, Q. Yu, R. Colby, D. Pandey, C. S. Park, J. Lian, D. Zemlyanov, I. Childres, V. Drachev, E. A. Stach, et al., Journal of Applied Physics 107, 044310 (pages 7) (2010).

4. J.-H. Chen, W. G. Cullen, C. Jang, M. S. Fuhrer, and E. D. Williams, Physical Review Letters 102, 236805 (2009).

5. G. Compagnini, F. Giannazzo, S. Sonde, V. Raineri, and E. Rimini, Carbon 47, 3201 (2009).

6. E. Stolyarova, D. Stolyarov, K. Bolotin, S. Ryu, L. Liu, K. T. Rim, M. Klima, M. Hybertsen, I. Pogorelsky, I. Pavlishin, et al., Nano Letters 9, 332 (2009).

7. F. Giannazzo, S. Sonde, V. Raineri, and E. Rimini, Applied Physics Letters 95, 263109 (2009).

8. L. Tapasztó, G. Dobrik, P. Nemes-Incze, G. Vertesy, P. Lambin, and L. P. Bir', Phys. Rev. B 78, 233407 (2008).

9. G. Kresse and J. Furthm $\square$ 1ler, Comput. Mat. Sci. 6, 15 (1996).

10. P. E. Blöchl, Phys. Rev. B 50, 17953 (1994).

11. J. P. Perdew, K. Burke, and M. Ernzerhof, Phys. Rev. Lett. 77, 3865 (1996).

12. B. Delley, The Journal of Chemical Physics 92, 508 (1990).

13. A. Zobelli, A. Gloter, C. P. Ewels, G. Seifert, and C. Colliex, Phys. Rev. B 75, 245402 (2007).

14. J. Kotakoski, A. V. Krasheninnikov, Y. C. Ma, A. S. Foster, K. Nordlund, and R. M. Nieminen, Physical Review B 71, 205408 (2005).

15. J. Kotakoski, A. Krasheninnikov, and K. Nordlund, Nucl. Instr. Meth. Phys. B 240, 810 (2005).

16. J. Kotakoski and K. Nordlund, New Journal of Physics 8, 115 (2006).

17. J. Kotakoski, A. V. Krasheninnikov, and K. Nordlund, Radiation Effects and Defects in Solids 162, 157 (2007). 
18. A. Tolvanen, J. Kotakoski, A. V. Krasheninnikov, and K. Nordlund, Applied Physics Letters 91, 173109 (2007).

19. D. W. Brenner, O. A. Shenderova, J. A. Harrison, S. J. Stuart, B. Ni, and S. B. Sinnott, J. Phys.: Condens. Matter 14, 783 (2002).

20. J. F. Ziegler, J. P. Biersack, and U. Littmark, The Stopping and Range of Ions in Matter (Pergamon, USA, 1985).

21. H. J. C. Berendsen, J. P. M. Postma, W. F. van Gunsteren, A. DiNola, and J. R. Haak, J. Chem. Phys. 81, 3684 (1984).

22. O. Lehtinen, J. Kotakoski, A. Krasheninnikov, A. Tolvanen, K. Nordlund, and J. Keinonen, Phys. Rev. B 81, in press (2010).

23. G. Hobler, Radiation Effects and Defects in Solids 139, 21 (1996).

24. A. V. Krasheninnikov and K. Nordlund, Phys. Rev. B 71, 245408 (2005). 